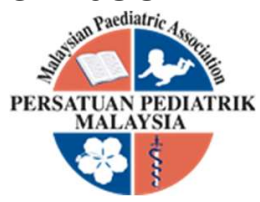

\title{
AN OVERVIEW OF PAEDIATRIC LIVER TRANSPLANT IN HOSPITAL SELAYANG: 2002 to 2015
}

\author{
Choy-Chen Kam ${ }^{1}$, Chooi-Bee Lim²
}

\begin{abstract}
Introduction: Hospital Selayang started the liver transplant program in 2002. We report a total of 81 liver transplant cases until year 2015. Among these, paediatric cases constitute almost half. This report aims to review the demography and outcome of paediatric liver transplant cases. Methodology: Case notes of all paediatric patients underwent liver transplant from year 2002 to 2015 were retrospectively reviewed. Results: A total of 38 paediatric cases received liver transplantations from year 2002 to 2015 . Age at transplantation ranged from 11 months to 16 years old (mean age 6 years) and weight ranged from 6.47 to 63 kilogram (mean 18kg). There were 20 males and 18 females, 20 of them were Malay, whereas Chinese and Indian were 13 and 4 respectively. Eighteen cases were living-related and 20 cases were cadaveric liver transplants. For recipient blood group, $\mathrm{O}+$ is the commonest. Biliary atresia was the most common indication for liver transplant (22 cases; $58 \%$ ), followed by intrahepatic cholestasis disorders (5 cases; $13 \%)$ and metabolic disease (4 cases; $10 \%)$. Post transplantation, there were $6(16 \%)$ biliary complications, 12 (32\%) vascular complications, 26 (68\%) developed early onset infection, $13 \%$ had acute rejection and 2 graft failure. Out of the 38 transplants, 79\% of them survive after 1 year, and $69.7 \%$ survive after 5 years. The common causes of mortality were sepsis and vascular complications. Conclusion: Despite limited resources, a successful paediatric liver transplant programme has been established in Hospital Selayang with good survival rate.
\end{abstract}

Keywords: Paediatric Liver Transplant, Hospital Selayang Malaysia, Liver Transplant Outcome DOI: $10.51407 /$ mjpch.v27i2.132

\section{Introduction}

Liver transplantation is a well-established treatment for acute or chronic liver failure and offers the opportunity for long healthy life with 20 -year survival rate $>80 \%$. Over the decades, the survival rate post liver transplantation has improved tremendously with the improvement of pre-transplant management, surgical technique, and prevention of opportunistic infection as well as the discovery and usage of potent immunosuppressive agents such as the calcineurin inhibitors.

The first liver transplant done in Malaysia was in 1993 in Subang Jaya Medical Centre which is a private institution. In year 2000, Hospital Selayang under the Ministry of Health Malaysia, was built as
Received: 1 January 2021; Accepted revised

manuscript: 2 August 2021

Published online: 17 October 2021 a tertiary liver centre as well as to initiate the national liver transplant program [1]. The first liver transplant done in Hospital Selayang was a paediatric case, performed on $10^{\text {th }}$ April 2002.

From year 2002 to 2015, a total of 38 cases paediatric liver transplant has been performed in Hospital Selayang. Unfortunately, the national paediatric liver transplant program was on hold since 2015 due to various factors. During this

'Hospital Tunku Azizah, (Hospital Wanita dan Kanak-kanak Kuala Lumpur), Jalan Raja Muda Abdul Aziz,Kampung Baru,50300 Kuala Lumpur, Malaysia

${ }^{2}$ Hospital Selayang,Lebuhraya Selayang-Kepong, 68100 Batu Caves Selangor Darul Ehsan, Malaysia

Corresponding Author:

Choy-Chen Kam, Hospital Tunku Azizah (Hospital Wanita dan Kanak-kanak Kuala Lumpur), Jalan Raja Muda Abdul Aziz, Kampung Baru,50300 Kuala Lumpur, Malaysia

Email: kcc797@gmail.com 
period patient has sought treatment in overseas centres. In year 2019, University Malaya Medical Centre has emerged as a new liver transplant centre in Malaysia. To date this centre has performed 13 cases of liver transplantation with good survival rate. There was a total of 78 cases paediatric liver transplantation were carried out from year 2014 to 2020 in oversea centres and University Malaya Medical Centre (unpublished data).

In view of the high demand of the service, Ministry of Health has decided to revive the national paediatric liver transplant program and shifted the service to Hospital Tunku Azizah (also known as Women and Children Hospital Kuala Lumpur). On 19 $19^{\text {th }}$ December 2020, the first paediatric liver transplant was carried out and this has marked another milestone. To date, Hospital Tunku Azizah and University Malaya Medical Centre are the two hospitals in Malaysia that provide paediatric liver transplantation service.

This study aims to review the demography and outcome of paediatric liver transplant cases performed in Hospital Selayang, as a historical record for future reference.

\section{Methodology}

This study is a retrospective record review. Case notes of all paediatric liver transplant performed from 2002 to 2015 were retrospectively reviewed.
The data collected included patients' demography, blood group, graft type, primary disease, morbidity and the causes of mortality, and was analysed using Statistical Package for Social Sciences (SPSS) version 23.0. Survival probabilities was estimated and compared between living-related and cadaveric liver transplant with Kaplan-Meier analysis. This study has received ethical approval from Medical Research and Ethics Committee [Ref No: NMRR17-1226-36331(IIR)].

\section{Results}

There were 38 children who underwent liver transplants from year 2002 to 2015, 20 (53\%) of whom were male. Age at transplantation ranged from 11 months to 16 years old (mean age 6 years) and weight ranged from 6.47 to 63 kilogram (mean 18kg). There were 20 males and 18 females. Twenty of them were Malay, whereas Chinese and Indian were 13 and 4 respectively. For recipient blood group, $\mathrm{O}+$ is the commonest, 13 cases, followed by $11 \mathrm{~B}+, 10 \mathrm{~A}+, 3 \mathrm{AB}+$ and $1 \mathrm{~B}$ . Eighteen cases (47.4\%) were living-donor liver transplants (LDLT) (56\% of whom the donor was the child's father).

Indications for liver transplant are shown in Table I. Biliary atresia was the most common indication for liver transplant (22 cases; 58\%), followed by intrahepatic cholestasis disorders (5 cases; $13 \%$ ) and metabolic disease (4 cases; $10 \%$ ).

Table 1. Causes of primary disease for liver transplant

\begin{tabular}{ll}
\hline Primary disease & No. of cases (\%) \\
\hline Biliary atresia & $22(58)$ \\
Alagille syndrome & $3(7.9)$ \\
Progressive familial intrahepatic cholestasis & $2(5.3)$ \\
Wilson's disease & $2(5.3)$ \\
Tyrosinaemia & $2(5.3)$ \\
Cryptogenic cirrhosis & $2(5.3)$ \\
Acute liver failure secondary to toxin & $2(5.3)$ \\
Autoimmune hepatitis & $1(2.6)$ \\
Primary sclerosing cholangitis & $1(2.6)$ \\
Sclerosing cholangitis secondary to Langerhans Cell Histiocytosis & $1(2.6)$ \\
\hline
\end{tabular}


This study mainly focuses on the immediate complications post transplantation. The main medical complications include infection (68\%), acute rejection (13\%) and graft failure (5\%). Surgical complications include vascular problems (24\%) and biliary tract problems (16\%) (Table 2). Twenty-four patients (68\%) experienced infections. There were a total of 42 infectious episodes in the 38 liver transplants (1.1 episode per patient), most of which were bacterial (78.6\%) and viral (19\%). Cytomegalovirus infection is the most common infection $(n=8)$. Interestingly, 5 out of the 8 patients were having new infection. All of the 38 patients received acyclovir as CMV prophylaxis. Acinetobacter baumannii and Klebsiella pneumoniae are the 2 significant bacterial infection that contributed to patient mortality (Figure 1). The common sources of infection are from the abdominal drain and also septicaemia.

Table 2. Post liver transplant complications

\begin{tabular}{lc}
\hline Events & No. of cases (\%) \\
\hline Biliary stricture & $6(16 \%)$ \\
\hline Vascular problem & $9(24 \%)$ \\
\hline Portal vein thrombosis & 4 \\
Portal vein stenosis & 3 \\
Hepatic artery thrombosis & 2 \\
Hepatic vein stenosis & 2 \\
Hepatic vein thrombosis & 1 \\
\hline Early onset infections & $26(68 \%)$ \\
Acute rejection & $5(13 \%)$ \\
Primary graft failure & $2(5 \%)$ \\
\hline
\end{tabular}

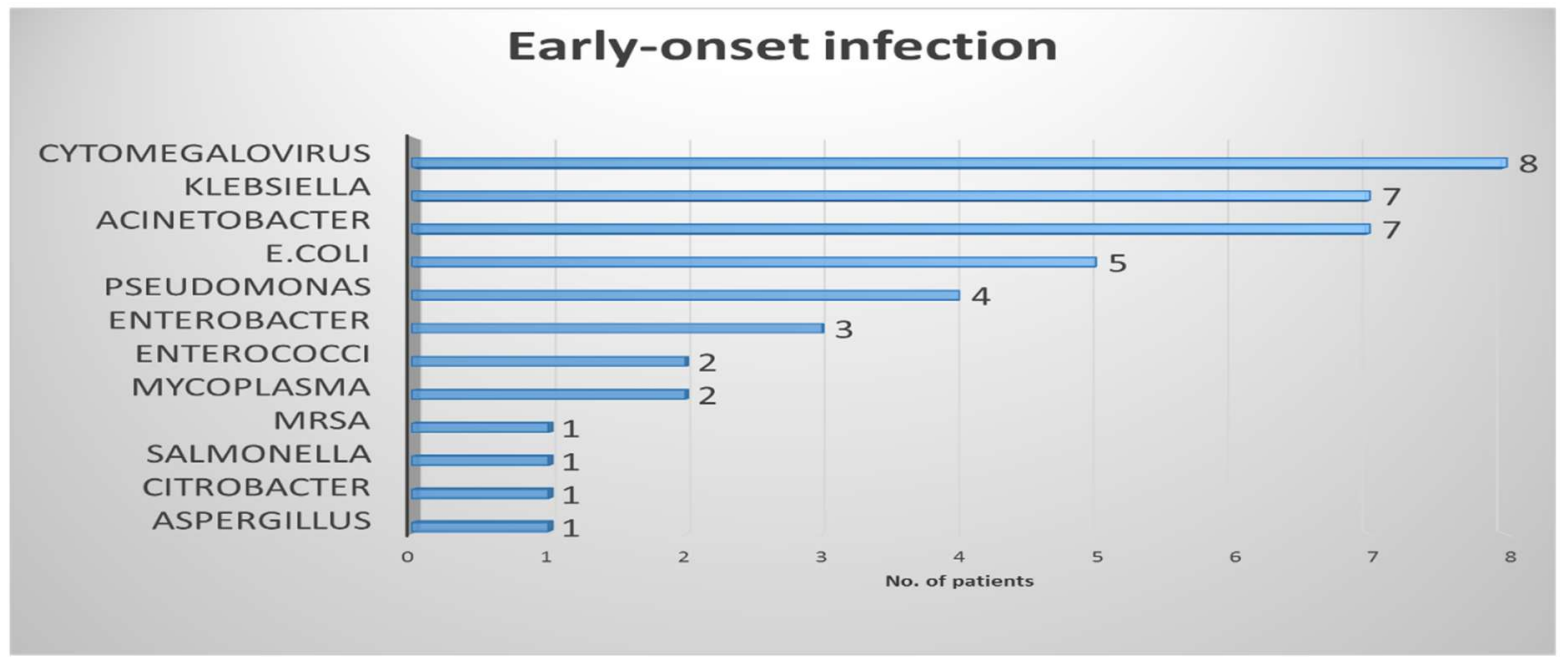

Figure 1. Organisms causing early-onset infection in post liver transplant patients

Vascular complications were encountered in 9 (24\%) patients, with portal vein thrombosis being the most common (4 patients), portal vein stenosis in 3 patients, hepatic artery thrombosis and stenosis in 2 patients respectively and hepatic vein thrombosis in one. Among these, 3 patients died of portal vein thrombosis.
The immunosuppressive protocol was designed with reference to protocols from various established liver transplant centres. Initially, patients were immunosuppressed using all three of methylprednisolone, Azathioprine and Tacrolimus. Prednisolone and Azathioprine were to be slowly weaned off leaving Tacrolimus as the 
single immunosuppressive therapy. The protocol was changed in late 2008 when Prednisolone and Tacrolimus remained, Azathioprine was replaced with mycophenolate mofetil (MMF). Patients were later on put on double immunosuppressants consists of Tacrolimus and MMF when Prednisolone slowly weaned off after 6 months post liver transplant.

The one- and five-year overall survival probability was $79 \%$ and $69.7 \%$ respectively (Figure 2 ), with no statistically significant difference between recipients of LDLT or deceased-donor liver transplants (DDLT) $(p=0.67)$ (Figure 3). There was no statistically significant difference of survival probability between age, gender or ethnic. Until year 2015, 28 (73.7\%) among 38 cases survived. The main cause of mortality is sepsis $(40 \%)$, where
Acinetobacter baumannii being the most common causative agent. Vascular complication (30\%) is the second leading cause of mortality post liver transplant where all 3 patients died of portal vein thrombosis. Two patients died of primary graft failure (20\%) and one from primary disease (recurrent Langerhans cells histiocytosis) (10\%).

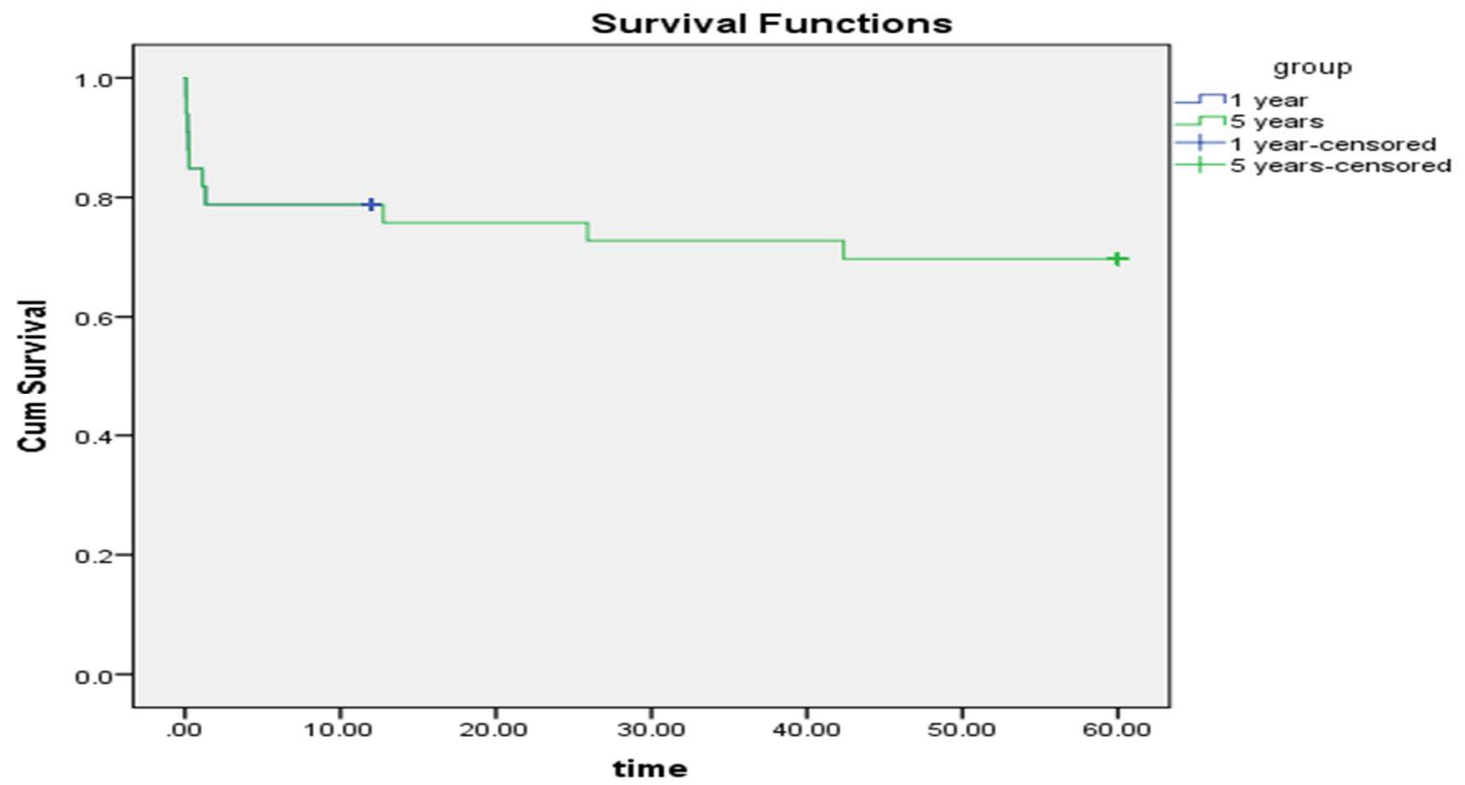

Figure 2. 1-year and 5-year survival rate post liver transplant 


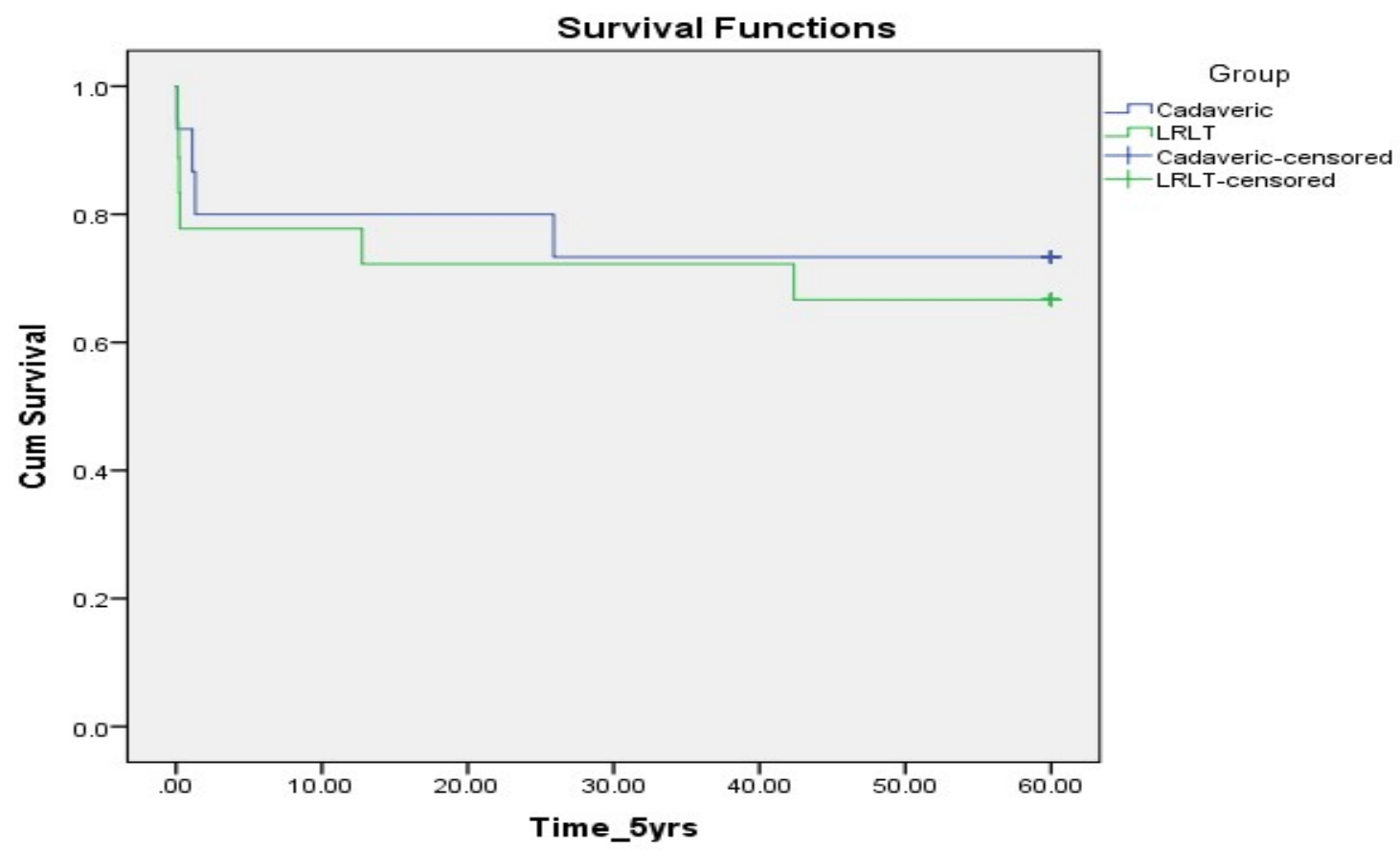

Figure 3. Comparison of survival rate between cadaveric and living-related liver transplant $(p=0.669)$

\section{Discussion}

Paediatric liver transplant (LT) outcome has improved over last 50 years with 20-year survival rate $>80 \%$. This results from improvement in surgical techniques as well as optimal use of immunosuppressive therapy that achieved a balance between minimising the risks of rejection and infection. Malaysia as a developing country encountered numerous problems in the initiation of the LT programme. This is a summary of paediatric LT programme in Hospital Selayang, the first hospital to carry out paediatric liver transplant under Ministry of Health Malaysia.

The overall 1-year survival probability $(78.8 \%)$ in this centre is lower compared with those reported in overseas centres. In Studies of Pediatric Liver Transplantation (SPLIT) registry which consist of data from the United States and Canada, 1-year survival probability for paediatric liver transplant was reported to be as high as $97.3 \%$ [2]. In United Kingdom the 1-year survival probability for children undergoing liver transplant is $95.7 \%$ [3]. Australia reported 1-year survival of $97 \%$ [4]. Slightly lower survival probability are found in Asian centres but comparable among each other, including Shanghai $91.36 \%$ at 1 year [5], Hong Kong $91 \%$ at 1 year [6], Taiwan $92 \%$ at 2 years [7] and Singapore: $85.7 \%$ at 1 year [8]. Our 5-year survival probability is also relatively lower compared with other centres (Malaysia 69.7\%; United Kingdom 91.8\%; Singapore 81.8\%; Hong Kong 88\%) $[3,6,8]$.

Studies have shown up to $80 \%$ of patients experience at least one infection episode within the first year of LT [9]. And some reported the rate of infection following liver transplantation is between $47 \%$ and $80 \%$, with a mean of one to 2.5 episodes of infections per patient [8]. In some centres, infections are the most common cause of mortality after LT. Underlying disease, malnutrition, immunosuppression and complexity of LT procedure are the major predispositions to infections [9]. The infection rate in our centre was as high as $68 \%$ and it was the main cause of mortality. CMV was the most common infection encountered in our cohort. The use of prophylactic anti-microbial agents such as ganciclovir for CMV disease was lacking in our centre and this has resulted in infectious complications. This inadequacy fortunately has been addressed as the access to the above medication has improved after the restart of paediatric liver transplant programme since year 2020 (at Hospital Tunku Azizah Kuala Lumpur). 
In terms of surgical complications, children suffered more surgical complications compared to adult, including vascular and biliary complications. Vascular complications following liver transplantation continue to be a significant cause of graft loss and patient morbidity. Vascular complications have a higher incidence in children (16\%-18\%) than adults (8\%-10\%) owning to the smaller vascular diameters [10]. Some has been reported as high as $25 \%$ to $30 \%$ in living-donor LT, paediatric LT and split deceased-donor LT [11]. The involvement of Plastic Surgery in connecting the vessels has improved the outcome. This is also true for biliary complications. The reported incidence of biliary complications after living donor LT is $10 \%$ to $35 \%$ in paediatric recipients [12]. Overall, our local experience is comparable to international and regional centre. Vascular complications were encountered in 9 (24\%) patients, with portal vein complications (stenosis and thrombosis) being the commonest involving 7 patients $(77.8 \%)$ and contributed to $30 \%$ of total mortality. Biliary complication is mainly caused by biliary hepaticojejunostomy stricture or biliary stricture. One patient required percutaneous transhepatic biliary drainage and subsequently succumbed due to sepsis.

The last paediatric liver transplant done in Hospital Selayang was 5 years ago in 2015. Many factors have contributed to the halt of this programme. The lack of awareness in nutritional optimisation has often led to severe faltering of growth and limited the chances of liver transplant. Delayed referral for liver transplant assessment also has resulted in situations where patients were too ill to be transplanted.

The lack of suitable living donor is yet another challenge, often due to absence of compatible blood group donor from patients' family, as well as high incidence of fatty liver disease and existing medical problem among potential donors. Further, paediatric patients are sharing the same pool with adult patients for the limited number of deceased liver donors.

On the other hand, the lack of resources including availability paediatric intensivist, paediatric intensive care unit beds, operation theatre and facilities are other difficulties the programme faced. Paediatric liver transplant recipients especially those with biliary atresia are mostly small-sized. Surgery in this group of patients is technically challenging and demands stable, highly skilled, experienced and adequately staffed surgical team which may not be always available in our public medical service. The other factor that may impact the development of local LT programme is: overseas liver transplant centres have become more popular in recent years and significant number of patients has chosen to be transplanted in those centres.

Multi-pronged efforts need to be undertaken to overcome the above challenges. LDLT using grafts donated by the patients' family is the commonest form of LT in Asia (90\% of LT's) due to religious and cultural beliefs, limited universal healthcare access in some countries, and lack of facilitative legislation [13]. We need to promote DDLT programme to increase the donor pool with the help of appropriate government policies. Besides, organ procurement agencies should be more proactive to approach patient and their families to educate them and increase the chance of obtaining consent for organ donation. The use of marginal livers, split-liver transplantation will expand the donor pool as well $[14,15]$. We also need to improve the awareness of optimisation of patient pretransplant management. It also goes without saying that general improvement of health care system and facilities is necessary for the LT programme to advance which includes but not limited to improvement of surgical skills, anaesthetic care, and intensive care.

In December 2020, Hospital Tunku Azizah Kuala Lumpur has performed the first paediatric liver transplant under Malaysia Ministry of Health again after 5 years. To date three liver transplants have been performed. Challenges are many and formidable, which call for more effort and contributions of all.

\section{Conclusion}

Despite limited resources, a successful paediatric liver transplant programme has been established in Hospital Selayang with good survival rate. We have made it before and we certain can make it again. It is hoped that Hospital Tunku Azizah is able to advance further from the past success of Hospital Selayang's LT programme. With greater resolve and commitment by all relevant stake holders, the programme can be revived to benefit more patients. 


\section{Acknowledgements}

We thank all medical, nursing, paramedic team who have contributed to the care of these children. We also thank Director General, Ministry of Health to give us permission to present this study.

\section{References}

[1] Thirteen report of the National Transplant Registry 2016, Malaysia.

[2] Elisofon SA, Magee JC, Ng VL, et al; for the Society of Pediatric Liver Transplantation Research Group. Society of pediatric liver transplantation: Current registry status 20112018. Pediatr Transplant. 2020; 24:e13605.

[3] Martin B, Ong EG. Paediatric liver transplantation: an overview. Paediatrics and Child Health. 2017 Dec 1; 27(12): 546-51.

[4] Stormon MO, Hardikar W, Evans HM, Hodgkinson P. Paediatric liver transplantation in Australia and New Zealand: 1985-2018. Journal of Paediatrics and Child Health. 2020 Nov; 56(11): 1739-46.

[5] Pan $Z Y$, Fan $Y C$, Wang $X Q$, et al. Pediatric living donor liver transplantation decade progress in Shanghai: Characteristics and risks factors of mortality. World J Gastroenterol. 2020; 26(12): 1352-64.

[6] Chan KL, Fan ST, Lo CM, William Wei I, Raymond Ng WM, Chung HY, et al. Paediatric liver transplantation in Hong Kong - a domain with scarce deceased donor. J Pediatr Surg. 2009 Dec 1; 44(12): 2316-21.

[7] Chen $C L$, Chen YS, Chiang YC, Cheng YF, Huang TL, Eng HL. Paediatric liver transplantation: a 10-year experience in
Taiwan. J Gastroenterol Hepatol. 1996 May; 11(5): S1-3.

[8] Aw M, Phua KB, Ooi BC, Da Costa M, Loh DL, Mak K, Tan KC, Isaac J, Prabhakaran K, Quak $\mathrm{SH}$. Outcome of liver transplantation for children with liver disease. Singapore Med J 2006; 47(7): 595-8.

[9] Selimoğlu MA, Kaya S, Güngör Ş, Varol Fi, Bağ HG, YIImaz S. Infection risk after paediatric liver transplantation. The Turkish Journal of Pediatrics 2020; 62: 46-52.

[10] Mali VP, Aw M, Quak SH, Loh DL, Prabhakaran K. Vascular complications in pediatric liver transplantation; single-center experience from Singapore. Transplantation Proceedings, 2012; 44(5): 1373-8.

[11] Astarcıoglu I, Egeli T, Gulcu A, Ozbilgin M, Agalar C, Cesmeli EB, Kaya E, Karademir S, Unek T. Vascular complications after liver transplantation. Exp Clin Transplant. 2019 Mar 14.

[12] Yukihiro S, Takumi K, Yuta H, Naoya Y, Noriki $\mathrm{O}$, Yoshiyuki I, et al. Biliary complications following pediatric living donor liver transplantation: Risk factors, treatments, and prognosis, transplantation. 2019; 103(9): 1863-70.

[13] Hibi T, Wei Chei AK, Chan A, Bhangui $P$, Current status of liver transplantation in Asia, International Journal of Surgery. 2020.

[14] Trotter JF. Expanding the donor pool for liver transplantation. Curr Gastroenterol Rep. 2000 Feb; 2(1): 46-54.

[15] deLemos AS and Vagefi PA. Expanding the donor pool in liver transplantation: Extended criteria donors. Clinical Liver Disease. 2013; 2: 156-9. 